\title{
REAL TIME SENSING IN THE NON LINEAR REGIME OF NEMS RESONATORS
}

\author{
Marc Sansa ${ }^{1,2}$, Van-Nghi Nguyen ${ }^{3}$, Sébastien Baguet ${ }^{3}$, Claude-Henri Lamarque ${ }^{4}$, Régis Dufour ${ }^{3}$ and \\ Sébastien Hentz ${ }^{1,2}$ \\ ${ }^{1}$ Université Grenoble Alpes, Grenoble, FRANCE \\ ${ }^{2}$ CEA, LETI, Minatec Campus, Grenoble, FRANCE \\ ${ }^{3}$ Université de Lyon, CNRS, INSA-Lyon, LaMCoS UMR5259, FRANCE \\ ${ }^{4}$ Université de Lyon, CNRS ENTPE/LGCB et LTDS, Vaulx-en-Velin, FRANCE
}

\begin{abstract}
This paper reports the proof of concept of a nonlinear detection scheme for Nanoelectromechanical (NEMS) resonators for sensing applications. This set-up increases the dynamic range of a resonant sensor by operating it at amplitudes beyond its limit of linearity. Unlike other works in non-linear sensing, this method allows the tracking of the resonance frequency in real time, while being suitable for multi-mode operation and hence, single-particle detection for example.
\end{abstract}

\section{INTRODUCTION}

Nanomechanical resonators have demonstrated their potential for a range of sensing applications, exhibiting unparalleled performance in mass or force sensing [1], [2] thanks to the miniaturization of the resonator. Up to now, in most works the resonators are operated in the linear regime, where the sensing resolution is limited by the dynamic range of the resonator [3]. Therefore, the dynamic range becomes a limiting factor of this technology, especially because the reduction of the resonators' dimensions implies a decrease of their dynamic range [4].

In order to avoid this limitation, it has been proposed to operate the resonator in the non-linear regime [5]-[7]. However, the complexity of the resonator's response in this regime presents a challenge: up to now, all proposed systems have to be "re-set" after each sensing event. This poses a serious limitation for real-time applications, where continuous detection within a specified bandwidth is required.

Nguyen et al. have recently proposed a theoretical detection technique in the nonlinear regime in real time [8]. Here we demonstrate the experimental feasibility of this method, based on the bifurcation of the response of the resonator when operated in non-linear regime.

\section{MEASUREMENT PRINCIPLE \\ NEMS resonators}

The experiments have been performed using crystalline silicon clamped-clamped beams, fabricated with hybrid DUV/e-beam lithography [9]. They feature a length of $10 \mu \mathrm{m}$, a width of $300 \mathrm{~nm}$ and a thickness of $160 \mathrm{~nm}$ (Figure 1). They are actuated electrostatically through a drive electrode, and their resonance frequency can be slightly tuned by applying a DC voltage to a second electrode nearby (labeled "shift" in Figure 1). The readout of the motion is piezoresistive, through two differential nanogauges located close to the clamping. Their resonance frequencies are typically $26 \mathrm{MHz}$ for the

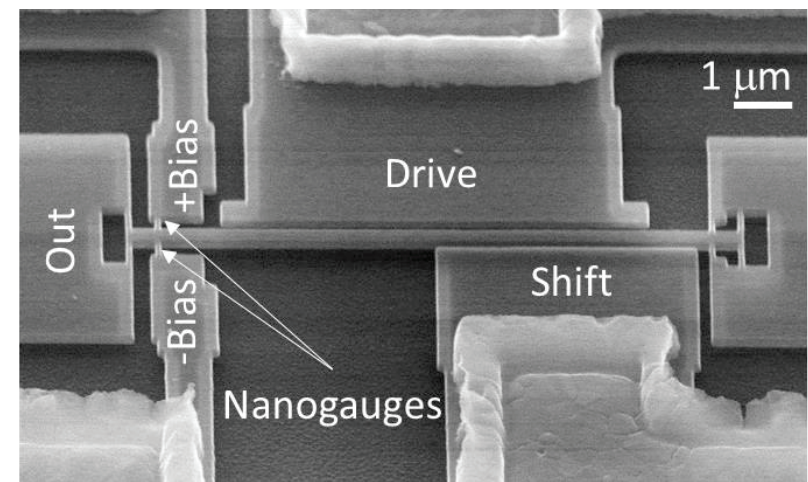

Figure 1: Crystalline silicon NEMS resonators. Clampedclamped beams with electrostatic actuation and piezoresistive detection.

first mode of resonance.

The resonators are measured using a down-mixing technique, in order to reduce the frequency of the readout signal. When the resonator is excited at a frequency $f_{0}$ using the drive electrode, the resistance of the nanogauges oscillates at the same frequency. Then, a voltage is introduced though the Bias electrodes at frequency $f_{0+} \Delta f$. The output signal is generated by the mixing between this voltage and the resistance producing a signal at frequency $\Delta f$, which can be arbitrarily low (typically in the range of tens of $\mathrm{kHz}$ ).

In terms of linearity, the resonators are limited by the stiffening of the beam at large amplitudes of motion, caused by the axial stretching of the resonator. This causes a so-called "hardening" non-linear behavior, with the resonance frequency increasing for large amplitudes of motion.

\section{Real-time non-linear measurement principle}

The non-linear measurement principle used here is discussed in detail in reference [8]. We operate the resonator in the nonlinear regime at specific points of its frequency response, close enough to the bifurcation point, with clear bistability. In the case of our resonators with hardening non-linearity, this means at the upper branch of the response, at frequencies higher than the linear resonance frequency. Then, a small shift down in resonance frequency brings about a large and abrupt jump-down in amplitude and causes the resonator to be operated in the monostable regime, as shown in Fig. 2a (in resonators with the softening non-linearity, the scenario for the jump should be adapted as described in 


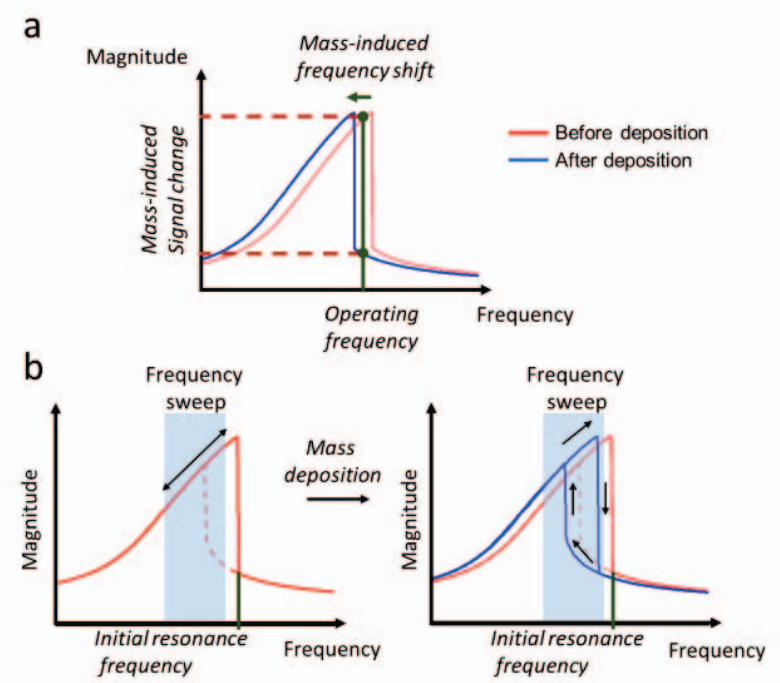

Figure 2: Real-time non-linear measurement principle. a) Non-linear measurement principle. Near the bifurcation point, small frequency shift causes an important change of the response of the resonator. b) Real-time measurement method, based on frequency-modulation.

reference [8]). The main disadvantage of this method is that it requires re-setting the resonator after each measurement: moving the measurement frequency down to the monostable regime, and up again to the initial position.

In order to avoid this issue, we propose to use a Frequency Modulation measurement method, similar to the ones used previously to electrically transduce the mechanical response of carbon nanotubes [10] or silicon nanowires [11]. With this set-up we continuously sweep up and down the response of the resonator, as shown in Figure $2 \mathrm{~b}$. Therefore, we obtain a real time measurement of the response of the resonator, both in a mono-stable (Figure 2b, left) or bi-stable (Figure 2b, right) regime, without a need to change the measurement conditions.

The implementation of this set-up is shown in Figure 3. The FM drive signal consists in a sweep signal $\phi_{F M}(t)$ at frequency $f_{M O D}$, which modulates the resonance frequency of the resonator $f_{0}$. In parallel, the same FM signal modulates the bias frequency $f_{0+} \Delta f$. The result is a downmixed signal independent of the modulation, appearing at frequency $\Delta f$, which can be chosen to be arbitrarily small. The output signal is then measured with a lock-in amplifier. In practice, we employ a low-frequency source to generate the FM-modulated signal, which is then fed to the RF sources generating the drive and bias signal: therefore, the complexity of this set-up is very similar to other down-mixing set-ups. Moreover, this measurement principle is adaptable to homodyne or heterodyne measurement techniques.

\section{Multi-mode operation}

Multi-mode operation is a requirement for NEMSmass spectrometry applications: the measurement of a single mode does not allow to deduce the mass and the position of a single particle deposited on the resonator. For clamped-clamped beams, the simultaneous

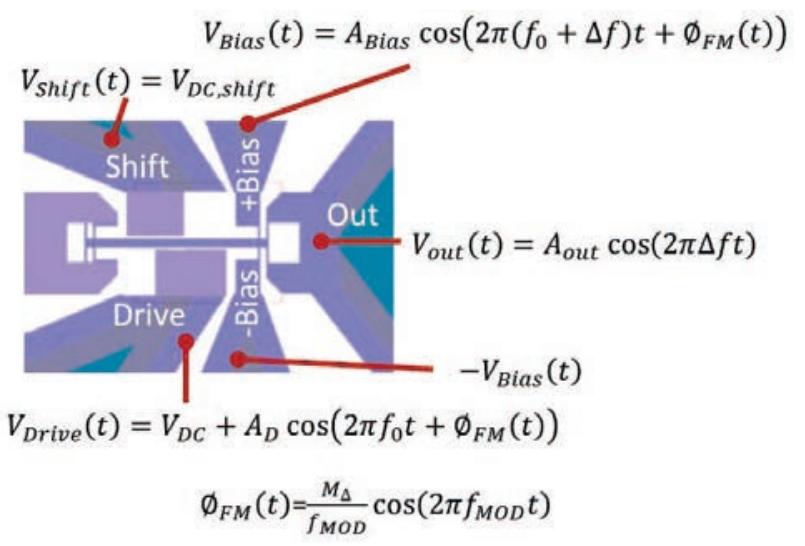

Figure 3: Frequency modulation measurement set-up. The same signal $\phi_{F M}(t)$ is used to modulate the frequency of the drive and bias signals. The result is a down-mixed measurement signal, independent of the modulation and at an arbitrary frequency $\Delta f . M_{\Delta}$ is the modulation index, which determines the span of the FM sweep.

measurement of the first and second resonance modes is necessary to deduce both parameters [12], [13]. At the same time, the simultaneous driving of the two modes implies a reduced dynamic range with respect to singlemode operation: the stretching of the resonator comes in this case from the addition of the stretching of both modes, and therefore the maximum linear amplitude of motion of each individual mode is decreased.

For this reason, the development of non-linear measurement techniques is especially important for NEMS mass spectrometry applications. The method that we show is adaptable to multi-mode operation by doubling each signal of the system: two different pairs of drive and bias voltages (one for each mode, at different $f_{0}$ ) are then needed. In this case, the information of each of the modes will be carried by two different measurement frequencies $\Delta f$, and therefore can be measured simultaneously with several lock-in amplifier channels.

\section{RESULTS}

In order to implement a proof of concept of the measurement technique, we first characterize the nonlinear behavior of the resonators. We measure a device setting the FM signal to $\phi_{F M}(t)=0$, and we find that a drive voltage with $A_{D}=0.2 \mathrm{~V}$ and $V_{D C}=0.1 \mathrm{~V}$ represents the limit of linearity of the resonator. We then set $V_{D C}=0.6 \mathrm{~V}$ in order to be in a strongly non-linear regime (Figure 4). The non-linear response of the resonator can be modelled as a duffing oscillator under these conditions. In order to simulate frequency shifts induced by mass deposition events, we introduce a DC signal to the "shift" electrode near the resonator (Figure 3). This DC voltage does not change the amplitude of motion of the resonator, but it changes its stiffness and therefore induces a decrease of its resonance frequency (Figure 4). This effect is very similar to that of a mass landing on the resonator. By changing this voltage from 0 to $0.8 \mathrm{~V}$, we induce frequency shifts up to $2 \mathrm{kHz}$ (Figure 4, inset), which is equivalent to a mass of $\sim 150 \mathrm{ag}\left(1.5 \times 10^{-16} \mathrm{~g}\right)$ deposited at the center of the beam. 


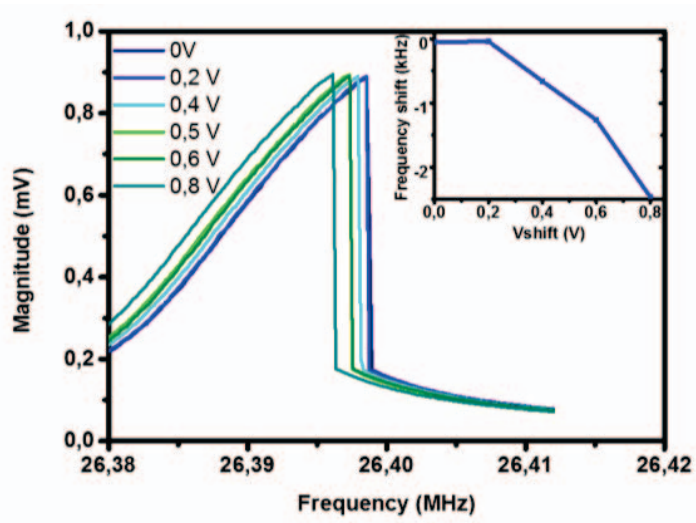

Figure 4: Non-linear response of the resonator. Frequency spectrum as a function of a DC voltage in the

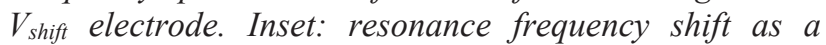
function of DC voltage. A shift of $2 \mathrm{kHz}$ corresponds to a mass or 150 ag deposited at the center of the resonator.

\section{Real-time measurement of the resonance frequency}

The FM measurement set-up is configured as follows. First, we set the FM modulation signal $\phi_{F M}(t)$ to 0 , and we measure the response of the resonator. The range of the FM sweep is configured at frequencies below the bifurcation point, but close to it. Then we switch on $\phi_{F M}(t)$, and start recording the magnitude of the output signal in real time (Figure 5a). The span of the frequency modulation sweep is a threshold limiting the minimum and maximum detectable resonance frequency shifts, and here it is typically few $\mathrm{kHz}$.

In Figure 5a we observe the effect of a resonance frequency shift in the output signal. In the initial conditions $\left(V_{\text {shift }}=0.4 \mathrm{~V}\right)$ the output signal does not reach the bifurcation point, and the output shows a smooth response. When a frequency shift is induced ( $V_{\text {shift }}=0.6$ $\mathrm{V})$ the sweep covers the bistable response of the resonator (Figure 2, blue curve): on its way up, is goes through the upper branch of the bistability until it reaches the bifurcation point (B1 in Figure 5a) and the monostable regime. On its way down, it goes through the lower branch of the response, until it reaches the other bifurcation point ( $B 2$ in Figure $5 \mathrm{a}$ ) and the monostable regime again. Each bifurcation point represents a point of infinite slope in the response, and therefore a jump in the magnitude of the measured signal. We observe this process as a function of time in Figure 5a.

In order to use this measurement technique in mass sensing, it is important to be able to precisely quantify the frequency shift in real time. In Figure $5 \mathrm{~b}$ we measure the response as a function of different frequency shifts induced every $\sim 300$ milliseconds by changing the DC voltage $V_{\text {shift }}$. We observe that the shape of the response changes in real time as a function of $V_{\text {shift. }}$. More concretely, we can define a parameter $\Delta W$, which corresponds to the difference between the two minima of the response (shown in Figure 5a). From the measurement of this parameter on each cycle of the FM sweep we can deduce the resonance frequency shift, as observed in Figure $5 \mathrm{~b}$. We demonstrated the detection of frequency shifts of $200 \mathrm{~Hz}$, corresponding to the deposition of a a

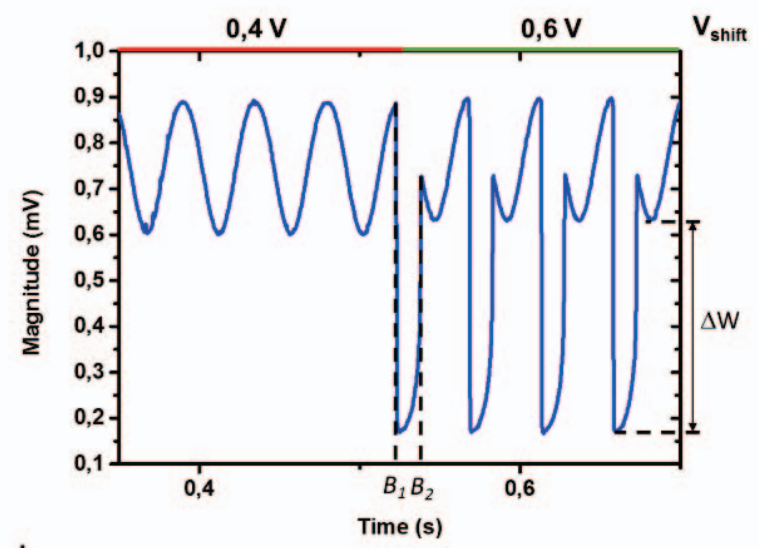

b

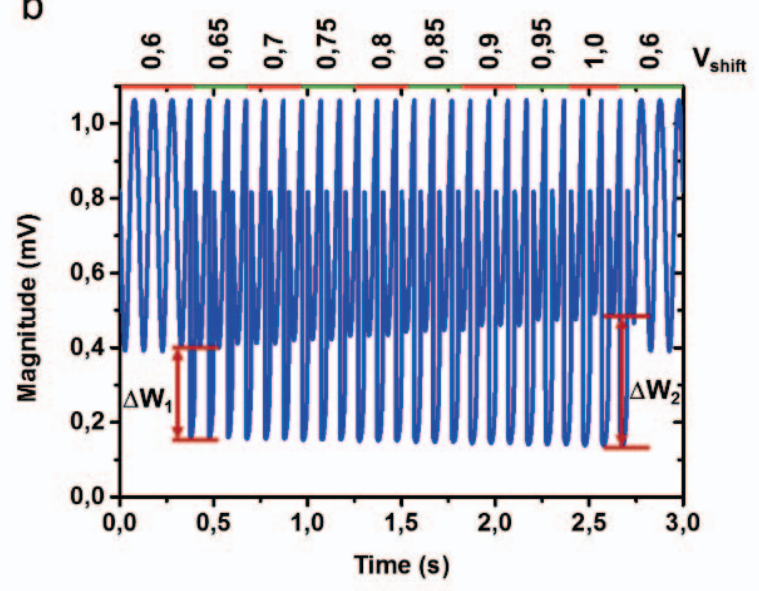

Figure 5: Real-time measurement of resonance frequency shifts: magnitude of the measurement signal at frequency $\Delta f$ as a function of time. a) Shape of the response as a function of a frequency shift induced by the $V_{\text {shift }}$ voltage. After the frequency shift the response goes through the two bifurcation points $B 1$ and $B 2$, resulting in abrupt shifts in the output signal. $\Delta W$ represents the difference between the two minima of the response, and can be used to quantify the frequency shift. b) Output signal as a function of frequency shifts induced every $300 \mathrm{~ms}$, with $f_{M O D}=100 \mathrm{~Hz}$.

mass of roughly $15 \mathrm{ag}$ at the center of the beam, with a modulation frequency $f_{M O D}$ of $89 \mathrm{~Hz}$.

The temporal resolution of the measurement technique is limited by the frequency of the FM signal, $f_{M O D}$. With the current configuration, this frequency is limited by the measurement set-up to roughly $250 \mathrm{~Hz}$, corresponding to a temporal resolution of $4 \mathrm{~ms}$. This is more than adequate for mass sensing applications, where the measurement time is usually in the range of tens of milliseconds to seconds [13]. Moreover, as usual, there is a trade-off between the response time of the system and its resolution: a faster $f_{M O D}$ requires a shorter measurement time, and therefore provokes an increased uncertainty in the measurement.

\section{CONCLUSIONS}

We showed a new mass sensing technique in realtime for NEMS resonators using their non-linear behavior. This scheme allows an increased signal level 
with respect to operation in the linear regime. In contrast to other non-linear methods, it does not require re-setting the system after each sensing event, therefore providing real-time measurement capabilities. Moreover, the set-up is adaptable to multi-mode operation. Both characteristics are important requirements for NEMS mass spectrometry applications.

We implemented this technique and demonstrated that it is suitable to track the resonance frequency in real time. The current set-up presents a response time of $4 \mathrm{~ms}$, while we demonstrated the detection of frequency shifts of $200 \mathrm{~Hz}$, corresponding to a deposited mass of $15 \mathrm{ag}$.

\section{REFERENCES}

[1] J. Chaste, A. Eichler, J. Moser, G. Ceballos, R. Rurali, and A. Bachtold, "A nanomechanical mass sensor with yoctogram resolution," Nat. Nanotechnol., vol. 7, no. 5, pp. 301-304, Apr. 2012.

[2] J. Moser, J. Güttinger, A. Eichler, M. J. Esplandiu, D. E. Liu, M. I. Dykman, and A. Bachtold, "Ultrasensitive force detection with a nanotube mechanical resonator," Nat. Nanotechnol., vol. 8, no. 7, pp. 493-496, Jul. 2013.

[3] A. N. Cleland and M. L. Roukes, "Noise processes in nanomechanical resonators," J. Appl. Phys., vol. 92, no. 5, pp. 2758-2769, Sep. 2002.

[4] H. W. C. Postma, I. Kozinsky, A. Husain, and M. L. Roukes, "Dynamic range of nanotube- and nanowire-based electromechanical systems," Appl. Phys. Lett., vol. 86, no. 22, pp. 223105223105-3, May 2005.

[5] V. Kumar, J. W. Boley, Y. Yang, H. Ekowaluyo, J. K. Miller, G. T.-C. Chiu, and J. F. Rhoads, "Bifurcation-based mass sensing using piezoelectrically-actuated microcantilevers," Appl. Phys. Lett., vol. 98, no. 15, p. 153510, Apr. 2011.

[6] R. L. Harne and K. W. Wang, "A bifurcationbased coupled linear-bistable system for microscale mass sensing," J. Sound Vib., vol. 333, no. 8, pp. 2241-2252, Apr. 2014.

[7] W. J. Venstra, M. J. Capener, and S. R. Elliott, "Nanomechanical gas sensing with nonlinear resonant cantilevers," Nanotechnology, vol. 25, no. 42, p. 425501 , Oct. 2014.

[8] V.-N. Nguyen, S. Baguet, C.-H. Lamarque, and R. Dufour, "Bifurcation-based micro/nanoelectromechanical mass detection," Nonlinear Dyn., vol. 79, no. 1, pp. 647-662, Nov. 2014.

[9] E. Mile, G. Jourdan, I. Bargatin, S. Labarthe, C. Marcoux, P. Andreucci, S. Hentz, C. Kharrat, E.
Colinet, and L. Duraffourg, "In-plane nanoelectromechanical resonators based on silicon nanowire piezoresistive detection," Nanotechnology, vol. 21, no. 16, p. 165504, Apr. 2010.

[10] V. Gouttenoire, T. Barois, S. Perisanu, J.-L. Leclercq, S. T. Purcell, P. Vincent, and A. Ayari, "Digital and FM Demodulation of a Doubly Clamped Single-Walled Carbon-Nanotube Oscillator: Towards a Nanotube Cell Phone," Small, vol. 6, no. 9, pp. 1060-1065, 2010.

[11] M. Sansa, M. Fernández-Regúlez, J. Llobet, Á. San Paulo, and F. Pérez-Murano, "Highsensitivity linear piezoresistive transduction for nanomechanical beam resonators," Nat. Commun., vol. 5, no. 4313, Jul. 2014.

[12] M. S. Hanay, S. Kelber, A. K. Naik, D. Chi, S. Hentz, E. C. Bullard, E. Colinet, L. Duraffourg, and M. L. Roukes, "Single-protein nanomechanical mass spectrometry in real time," Nat. Nanotechnol., vol. 7, no. 9, pp. 602-608, Sep. 2012.

[13] E. Sage, A. Brenac, T. Alava, R. Morel, C. Dupré, M. S. Hanay, M. L. Roukes, L. Duraffourg, C. Masselon, and S. Hentz, "Neutral particle mass spectrometry with nanomechanical systems," Nat. Commun., vol. 6, p. 6482, Nov. 2015.

\section{ACKNOWLEDGEMENTS}

The authors are grateful for the support from the LETI Carnot Institute NEMS-MS project, as well as the I@L/LETI Intern-Carnot NEMROD project and the Marie-Curie Eurotalents ingoing fellowship (M.S.).

\section{CONTACT}

*Sébastien Hentz; sebastien.hentz@cea.fr 